\title{
Penile Injection Therapy
}

National Cancer Institute

\section{Source}

National Cancer Institute. Penile Injection Therapy. NCI Thesaurus. Code C107635.

Self-administered injections of vasodilators into the corpus cavernosa to promote engorgement and tumescence of the glans with sexual stimulation. 